\title{
Insulinmobilisierung beim Hund durch Scheinfütterung mit Glukose ${ }^{1}$
}

Aus Untersuchungen an verschiedenen Spezies ${ }^{2-4}$ ist bekannt, dass der Anstieg der Insulinkonzentration im peripheren Venenblut nach peroraler Glukoseverabfolgung wesentlich grösser ist, als bei Wahl des intravenösen Weges, obwohl nicht annähernd so hohe Blutzuckerwerte erreicht werden. Es liegt nahe, für diesen Unterschied die insulinmobilisierende Wirkung der Enterohormone wie Sekretin, Pankreozymin oder Enteroglukagon als Ursache zu betrachten ${ }^{5,6}$. Es ist noch nicht sicher, ob die physiologischen Konzentrationsschwankungen dieser Substanzen auch in vivo für die Insulinmobilisierung von Bedeutung sind. Auch wurde eine Steuerung uber den N. vagus wahrscheinlich gemacht ${ }^{7-9}$. Wir haben überprüft, inwieweit schon eine Scheinfütterung mit Glukose zur Insulinausschüttung führt.

Methodik. Schäferhundbastarde beiderlei Geschlechts im Alter von $26 \pm 5$ Monaten (Körpergewicht 21,2士 $0,9 \mathrm{~kg}$ ) wurden über 3-6 Wochen systematisch an die Versuchsbedingungen adaptiert. Dann legten wir ihnen im Halsbereich eine doppelläufige Ósophagusfistel an, die bei peroraler Verabfolgung von Testlösungen deren nahezu vollständige Wiedergewinnung an der oralen Fistelöffnung erlaubt. Frühestens 2 Wochen post operationem erhielten die wachen Tiere nach 18stündiger Nahrungskarenz $1,0 \mathrm{~g} / \mathrm{kg}$ Glukose in $50 \mathrm{ml}$ Leitungswasser peroral verabfolgt. Bis $z u \quad 90 \mathrm{~min}$ wurden der Blutzucker ${ }^{10}$ und die Konzentration des radioimmunologisch reagierenden Insulins ${ }^{11}$ im peripheren Venenblut überprüft.

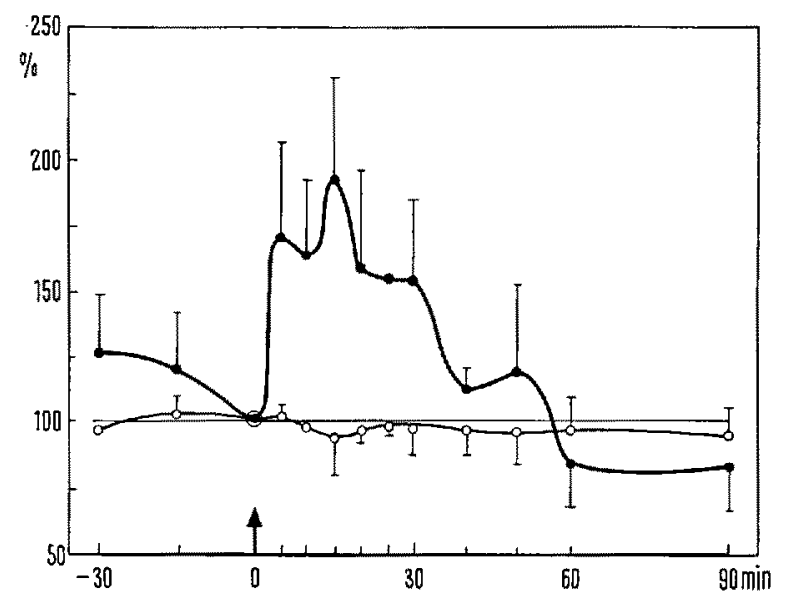

Glykämie und Insulinkonzentration (IRI) im peripheren Venenblut von wachen Hunden mit doppelläufigen Ốsophagusfisteln nach peroraler Gabe von $1,0 \mathrm{~g}$ Glukose $/ \mathrm{kg}(\uparrow)$ in $\%$ der unmittelbar vor Applikation gemessenen Werte $(n=10,100 \%$-Werte: Glykämie $77,9 \pm$ $1,1 \mathrm{mg} / 100 \mathrm{ml}$, IRI $30,5 \pm 6,5 \mu \mathrm{E} / \mathrm{ml})$.
Befunde und Diskussion. Aus der Figur geht hervor, dass in dieser Testanordnung der Blutzuckerspiegel nicht ansteigt. Dementsprechend konnten 93-97\% der applizierten Glukosemenge an der oralen Fistelöffnung wiedergewonnen werden. Trotzdem ist die Insulinkonzentration bereits nach $5 \mathrm{~min}$ statistisch sicher $(P<1 \%)$ erhöht. Dieser Anstieg setzt sich bis zur 30. min fort. Wegen der beträchtlichen Streubreite der Ergebnisse ist noch keine Aussage dariber möglich, ob den sich andeutenden verschiedenen Phasen des Insulinanstiegs eine Bedeutung zukommt. Weitere Untersuchungen in unserem Laboratorium lassen auch nach p.o. Applikation von Leitungswasser eine Insulinausschüttung erkennen.

Nach diesen Befunden ist es möglich, durch perorale Scheinfütterung mit Glukose eine Insulinfreisetzung zu provozieren, ohne dass ein messbarer Blutzuckeranstieg auftritt und ohne dass die Testlösung diejenigen Areale des Magen-Darm-Traktes erreicht, von denen aus die Mobilisierung von Enterohormonen ausgelöst wird. Es liegt nahe, einen eingeschliffenen bedingten Nahrungsreflex oder eine von der Mundhöhle aus reflektorisch vermittelte Hormonfreisetzung im Sinne einer Vorwärtskopplung zu vermuten.

Summary. In conscious, trained dogs with doublebarrelled fistulas of the oesophagus, an increase of the insulin concentration in the peripheral venous blood, without change of the glycaemia, was observed after sham-feeding with glucose.

U. FISCHER und H. HOMMEL

Zentralinstitut fïr Diabetes,

DDR-2201 Kanlsburg-Greifswald (DDR), 1. Oktober 1970.

1 Diese Untersuchungen wurden mit Mitteln eines Forschungsauftrages des Ministeriums für Gesundheitswesen der DDR durchgeführt.

* H. ElRick, L. Stimmter, C. J. HLAd JR. und Y. Arai, J. clin. Endocr. 24, 1076 (1964).

${ }^{8}$ M. J. Perley und D. M. KipNis, J. clin. Invest. 46, 1954 (1967).

4 M. S. NijJar und W. F. Perry, Diabetes 19, 155 (1970).

$5 \mathrm{~N}$. MCInTYre, C. D. Holdosworth und D. S. Turner, Lancet $1964 \mathrm{II}, 20$.

- R. H. Unger und A, M. Ersentraut, Arch. intern. Med. 123, 261 (1969).

7 L. A. Frohman, E. Z. EzdinLI and R. JAvid, Diabetes 16,443 (1967).

B A. Kaneto, K. Kosaka und N. Nakao, Endocrinology 80,530 (1967).

- G. Lagache, B. Combemale, J. C. Fourlinnie und M. Linguette, Acta gastroent. belg. 32, 87 (1969).

to P. Köhler, Z. ges, inn. Med. 17, 674 (1962).

11 C. N. Hayes und P. J. Randle, Biochem. J. 88, 137 (1963).

\section{Potassium Inactivation in Myelinated Nerve Fibres}

Recent experiments on various excitable tissues (e.g. squid axon ${ }^{1}$, frog muscle fibre ${ }^{2}$ ) have shown that the potassium permeability slowly inactivates if the membrane is kept depolarized. This process is not contained in the classical HoDGKIN-HuXLEY model ${ }^{3}$. For myelinated nerve fibres, a quantitative description of $\mathbf{K}$ inactivation was lacking. Therefore, we carried out voltage clamp experiments on isolated nerve fibres of the toad, Xenopus laevis, using the method of DoDGE and FrankenHAEUSER ${ }^{4}$. Upon a depolarization, the potassium current rapidly reached a peak value and then decreased almost exponentially to a stationary value, $I_{k \infty}$ (Figure 1). To show that this remaining current was indeed a potassium current, tetraethylammonium chloride (TEA), a specific inhibitor of the potassium permeability, was applied which further reduced the outward current to the level 